\title{
Síntesis y caracterización de materiales compuestos por Ti-HAP y Ti-HAP-Poros para aplicaciones como biomateriales
}

\section{Synthesis and characterization of Ti-HAP and Ti-HAP-Pores composite materials for biomaterials applications}

Presentación: 6-7/10/2020

\section{Doctorando:}

\section{Santiago Marcelo Cantero}

Ingeniería Metalúrgica, Facultad Regional Córdoba, Universidad Tecnológica Nacional - Argentina. santiagomcantero@hotmail.com

\section{Director/a:}

\section{Carlos Oldani}

\section{Co-director/a:}

\section{Roberto Oscar Lucci}

\section{Resumen}

Este trabajo investiga dos procesos de obtención de materiales compuestos de Ti-HAP e Ti-HAP-Poros mediante procesos pulvimetalúrgicos. Las muestras "en verde" son conseguidas por compactación para el primer proceso y por aplicación de la técnica de Gel-Casting para el segundo; en donde dichas muestras son posteriormente sinterizadas para obtener el material compuesto Ti-HAP o Ti-HAP-Poro. Los polvos utilizados son hidruro de titanio ( $\mathrm{TiH}_{2}$ ), hidroxiapatita (HAP) y resina acrílica (RA). Se realiza análisis por microscopía óptica (OM), microscopía electrónica de barrido (SEM) y espectroscopía de rayos X de energía dispersa (SEM-EDS) junto al análisis de imágenes mediante el software Matlab.

Palabras clave: Biomateriales, titanio, hidroxiapatita, sinterización, poros.

\begin{abstract}
This research is about the study of two different methods to obtain composed materials of Ti-HAP and Ti-HAP-Pores through pulvimetalurgy. On one hand, the "green samples" are obtained by mix powders compaction and on the other hand through Gel-Casting technique; with subsequent sinterization to obtain a composed material of Ti-HAP or Ti-HAPPore. Mix powders are composed by different amounts of titanium hydride $\left(\mathrm{TiH}_{2}\right)$, hydroxyapatite (HAP) and acrylic resin (RA). Optical microscopy (OM), scanning electron microscopy (SEM) and energy dispersed X-ray spectroscopy (SEM-EDS) analysis are made together with image analysis using Matlab software.
\end{abstract}

Keywords: Biomaterials, titanium, hydroxyapatite, sintering, pores. 


\section{Introducción}

Los implantes son elementos necesarios para reparar o alterar los tejidos corporales naturales. Para ello, se deben desarrollar de tal manera que sean "amigables" con el cuerpo humano y que cumplan con ser biocompatibles y poseer propiedades químicas y mecánicas para satisfacer la necesidad demandada por el cuerpo humano (Navarro et al., 2008). Para aplicaciones biomédicas el titanio se destaca debido a que presenta propiedades mecánicas adecuadas, es cuasibioinerte, es el único biometal que se oseointegra y posee una excelente resistencia a la corrosión (Shettlemore \& Bundy, 1999; Tengvall et al., 1989). Sin embargo, el Ti presenta cierta complejidad en el manejo de las técnicas de fundición y colada debido a su alta reactividad (Lascano, 2012); condición que implica la búsqueda de nuevas tecnologías de fabricación. Además, los metales poseen una excesiva rigidez frente al hueso (Oldani, 2014), pudiendo generar el fenómeno conocido como "Stress-Shielding" el cual promueve una enfermedad conocida como "osteoporosis" (Cantero \& Roure, 2017). Esto implica la búsqueda de alternativas que permitan reducir la rigidez de los materiales empleados. La rigidez del Ti puede reducirse mediante la adición de elementos de aleación, la modificación de la estructura metalográfica o la generación de estructuras porosas(Rack \& Qazi, 2006).

Existe un método pulvimetalúrgico (PM) para desarrollar estructuras porosas de Ti a través del método de gel-casting y sinterización (GCS). Investigaciones recientes muestran la posibilidad de aplicar el método de Gel-Casting con polvo de hidruro de titanio $\left(\mathrm{TiH}_{2}\right)$ y resina acrílica $(\mathrm{RA})$ para obtener muestras en "verde" mediante polimerización de la RA y posterior sinterización a altas temperaturas y atmósferas de alto vacío, con la obtención de estructuras con porosidad controlada. En el proceso de sinterización, la RA se volatiliza, contribuyendo con la formación de cavidades o poros en el material. Los principales resultados muestran que es posible obtener estructuras porosas con diferentes características de porosidad en función de la cantidad de RA empleada en la mezcla (Roure et al., 2016).

Por otra parte, la hidroxiapatita (HAP) es un material biocerámico con propiedades mecánicas deficientes, pero posee una estructura similar a los huesos con buenas propiedades bioactivas y biodegradables que pueden promover el crecimiento de tejidos naturales (Niinomi, 2008). Es posible la generación de un material compuesto de Ti e HAP, el cual pudiera englobar propiedades como ser de carácter bioinerte, bajo módulo de Young y alta biocompatibilidad (Cui et al., 2005; Niespodziana et al., 2010).

Por lo tanto, este trabajo investiga la obtención de materiales compuestos Ti-HAP y Ti-HAP-Poros mediante métodos pulvimetalúrgicos, estudiando las características químicas y microestructurales de los materiales obtenidos.

\section{Desarrollo}

Dos técnicas pulvimetalúrgicas, fueron utilizadas para la fabricación de las muestras en verde. La primera técnica consiste en la preparación de muestras mediante la mezcla de polvos y la compactación mecánica por simple efecto (CM), mediante prensado hidráulico a $1000 \mathrm{MPa}$ en moldes cilíndricos de $8 \mathrm{~mm}$ de diámetro. La mezcla se conforma de polvos de hidruro de titanio (II) de la compañía "Sigma Aldrich", tamaño de partícula inferior a los $44 \mu \mathrm{m}$ y pureza del $98 \%$; mezclada con hidroxiapatita en polvo de origen bovino (marca Tissum) con una granulometría menor a $200 \mu \mathrm{m}$. Las proporciones de las mezclas que se estudian son con 5 y $10 \%$ en peso de hidroxiapatita, y el restante formado por hidruro de titanio (II). La segunda técnica consiste en la preparación de muestras mediante el método de Gel-Casting (GC). Aquí se mezclan polvos de hidruro de titanio con mismas características que el utilizado en el método CM, polvos de resina acrílica compuestos por metacrilato de polimetilo $(88 \mu \mathrm{m})$ de la compañía "Cardent" e hidroxiapatita en polvo de origen sintético de la compañía "Sigma Aldrich". Se estudian mezclas con cantidades fijas de $12 \%$ de resina acrílica y cantidades de 5 y 10 $\%$ de hidroxiapatita. La mezcla de polvos es combinada con peróxido de benzoilo (iniciador de reacción) y metacrilato de metilo (monómero de secado automático), ambos en estado líquido. Esto permite obtener una solución viscosa o pasta, conocida como lodo o "slurry". Cuando la pasta consigue una viscosidad con suficiente fluidez, es vertida dentro de un molde de silicona con forma cilíndrica de $8 \mathrm{~mm}$ de diámetro y $12 \mathrm{~mm}$ de altura. Después de 24 horas, las muestras están 
secas (muestras en verde) y pueden desmoldarse. Para ambos casos, la mezcla de polvos se realiza en un mezclador de vidrio tipo "Y" durante un tiempo de 15 minutos a $34 \mathrm{rpm}$.

Las muestras en verde se llevan hacia la etapa de sinterización (CMS y GCS). La misma se realiza en un horno eléctrico tubular de alta temperatura fabricado por "Sentro Tech" el cual se encuentra conectado a una bomba de alto vacío marca "Edwards". El ciclo CMS consiste en dos etapas mientras que el ciclo GCS consiste en tres etapas:

I. Volatilización de RA: Esta etapa solo se lleva a cabo para el método GCS, la misma ocurre a una temperatura de $200{ }^{\circ} \mathrm{C}$ durante un tiempo de 30 minutos. A esta temperatura el polímero libera compuestos gaseosos como $\mathrm{H}_{2}$, $\mathrm{CO}_{2}$ y $\mathrm{CO}$, los que son eliminados a por la bomba de vacío. El objetivo de esta etapa es eliminar la RA que contiene la muestra en verde, generando espacios libres dentro del material, con el fin de obtener una estructura porosa.

II. Descomposición de partículas de $\mathrm{TiH}_{2}$ : En esta etapa, las muestras en verde se calientan hasta una temperatura de $550^{\circ} \mathrm{C}$ con una permanencia de 90 minutos para el método CMS y a $600^{\circ} \mathrm{C}$ durante 45 minutos para el método GCS. El $\mathrm{TiH}_{2}$ es transformado en Ti metálico e $\mathrm{H}_{2}$ gaseoso, el cual es eliminado, como se indica en la ecuación (1):

$$
\mathrm{TiH}_{2}(\mathrm{~s}) \rightarrow \mathrm{Ti}(\mathrm{s})+\mathrm{H}_{2}(\mathrm{~g})
$$

III. Sinterización de las partículas de Ti: En esta etapa, se logra la unión de las partículas de Ti a través de un proceso difusional en estado sólido. Este proceso es realizado con protección de atmósfera de vacío a $750{ }^{\circ} \mathrm{C}$ por un tiempo de 240 minutos para el método CMS y a $780 \stackrel{\circ}{\circ}$ con una duración de 420 minutos para el método GCS. Una vez finalizado el ciclo CMS, se obtiene un material compuesto de Ti-HAP, mientras que una vez finalizado el ciclo GCS, se obtiene un material compuesto de Ti-HAP-Poros.

Luego de obtener las muestras metálicas, se lleva a cabo un análisis mediante microscopía óptica (OM), microscopía electrónica de barrido (SEM-EDS) y análisis de imágenes por medio de un "scrib" en el software Matlab (The MathWorks Inc., vR2017a). Se caracteriza el tamaño, forma y distribución de poros, así como tamaño, forma y distribución de las partículas de HAP. Los resultados se procesan mediante análisis estadístico descriptivo en el software Minitab 18 (Minitab LLC., V18.1, EE. UU.)., donde las variables de interés fueron porosidad media (Pm), área media de las partículas de HAP $(\mathrm{Am})$, coeficiente de variación (CV), valor mínimo (Min) y valor máximo (Max) de los poros y partículas de HAP analizadas.

La porosidad o el área que ocupan las partículas de HAP en la superficie analizada, se calcula según la ecuación (2), Donde Ap es el área de los poros o partículas de HAP, según corresponda, y At es el área de la imagen metalográfica. Asumiendo que las partículas de HAP y los poros son esféricos, se calculó un diámetro equivalente (Deq) para cada uno de ellos, según la ecuación (3). Por último, el factor de forma (FF) se calcula para cada poro y cada partícula de HAP, mediante la ecuación (4), donde $\mathbf{d}_{\min }$ es el eje menor y $\mathbf{d}_{\max }$ es el eje mayor de una elipse que se ajustó al perímetro de los poros y partículas de HAP, mediante el criterio de mínimos cuadrados. El valor 0 (cero) corresponde a la máxima irregularidad, mientras que el valor 1 (uno) corresponde a una forma esférica:

$$
\mathrm{P}=\text { Ap } / \text { At } \quad(2) ; \quad \text { Deq }=(4 A p / \pi) 1 / 2 \quad(3) ; \quad F F=d_{\min } / d_{\max }
$$

También se realiza un análisis químico mediante SEM-EDS. El objetivo de este estudio es comprobar si existe un proceso de reacción o descomposición de $\mathrm{P}$ o Ca de las partículas de HAP, la cual puede ocurrir por encima de los $1000{ }^{\circ} \mathrm{C}$ en atmósferas normales y alrededor de los $800^{\circ} \mathrm{C}$ en vacío (Colazo \& Bocco, 2017). Se analiza químicamente al polvo de HAP previo al proceso de sinterizado y luego se lo compara con el resultante del proceso de sinterizado.

\section{Resultados y discusión}

Se corrobora la correcta sinterización tanto para el método CMS como GCS. La estructura metalográfica del método CMS (Figura 1 a), revela una matriz continua de Ti $(\alpha)$ interrumpida por las partículas de HAP (observadas en negro). Para el método GCS (Figura 1 b) se observa una correcta sinterización del titanio formando la matriz de Ti $(\alpha)$ interrumpida por poros (observados en negro) y por partículas de hidroxiapatita (observadas en gris oscuro). 


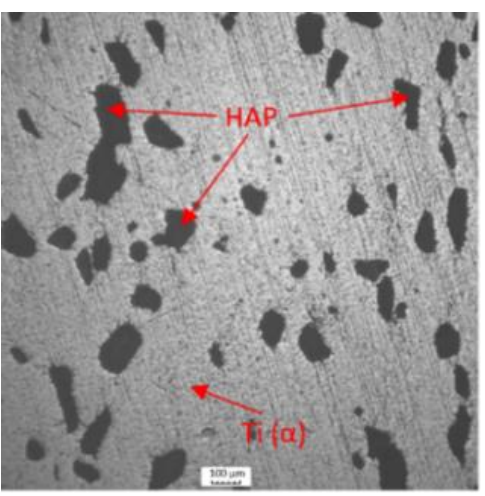

a)

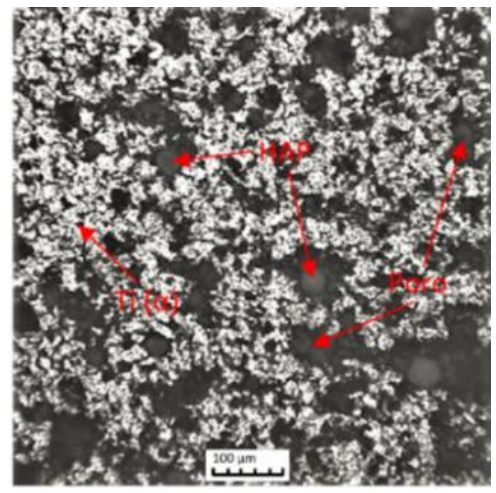

b)

Figura 1: Imágenes de la estructura metalográfica obtenidas por OM. a) Proceso CMS. b) Proceso GCS. Magnificación: 100 X.

El método GCS permite la obtención de estructuras porosas metálicas, cuyo análisis se muestra en la Figura 2. Se evidencia una porosidad promedio de $37 \%$ con un rango de tamaño variable de 4 a $114 \mu \mathrm{m}$ y diferentes formas de los poros (0,2 a 1). En la Figura 2a) podemos observar que la mayoría de los poros presentan un tamaño pequeño (10 a 30 $\mu \mathrm{m}$ ) los cuales son atribuidos a procesos difusionales en la sinterización y solo algunos poseen tamaños mayores (45 a 75 $\mu \mathrm{m})$ que son generados intencionalmente mediante la volatilización de la RA. En la Figura 2b) se puede observar que la forma que adoptan los poros es variada y no respetan una tendencia definida.

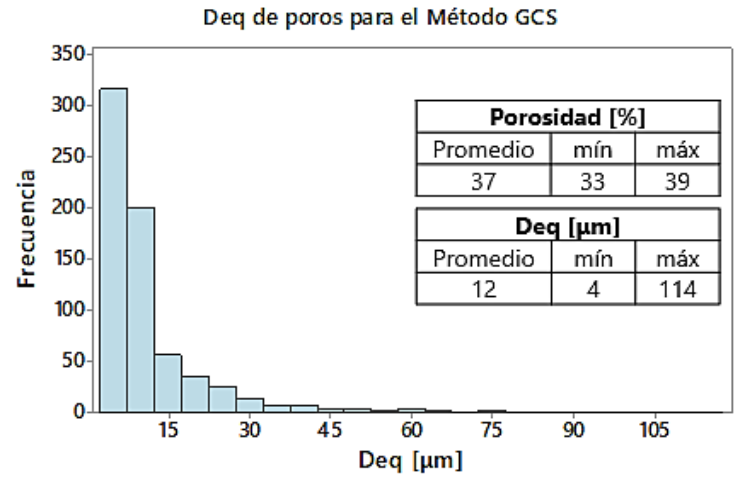

a)

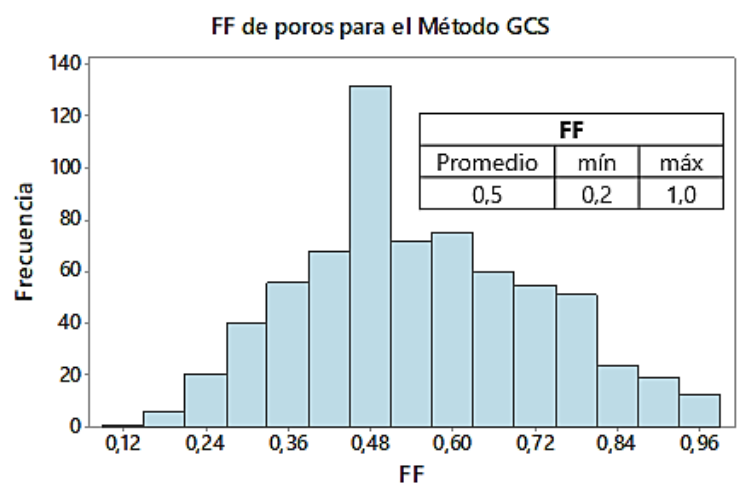

b)

Figura 2: Análisis estadístico e histogramas de distribución de tamaño y forma de poros para el proceso CGS. a) Deq. b) FF.

La porosidad y el tamaño de los poros tienen un papel importante en el correcto crecimiento óseo. Por un lado, la bibliografía sugiere que se necesitan porosidades de al menos $55 \%$ para obtener poros interconectados, los cuales promueven el crecimiento óseo hacia el interior de la estructura porosa (Bram et al., 2006; Esen \& Bor, 2007). Por otro lado, un crecimiento óseo óptimo depende de dos tipos de mecanismos diferentes: adhesión y migración celular. Estos mecanismos funcionan simultáneamente y cada uno se desarrolla mejor según sus propias condiciones. Para una óptima adhesión celular se recomienda un tamaño de poro entre 100 a $150 \mu \mathrm{m}$, mientras que para promover la migración celular se recomiendan tamaños de poros desde $300 \mu \mathrm{m}$ hasta $800 \mu \mathrm{m}$ (Murphy \& O’Brien, 2010). Los resultados obtenidos no alcanzan a los valores recomendados por la bibliografía, por lo que es necesario el estudio futuro de nuevas alternativas de mezcla de RA, en mayores cantidades y con partículas de tamaño mayores.

La Figura 3 muestra los resultados obtenidos del procesamiento de imágenes, correspondientes a las características medidas de la HAP bovina y sintética. Para ambas partículas de HAP podemos ver que las mismas no varían tanto en tamaño como en forma luego de la sinterización. La distribución de tamaño de la HAP bovina se ve en la Figura 3a $(<200$ $\mu \mathrm{m})$ y la correspondiente a la HAP sintética en la Figura $3 \mathrm{~b}(<30 \mu \mathrm{m})$. El FF también concuerda con la condición de 
irregularidad para la HAP bovina (Figura 3a), y de forma regular para la HAP sintética (Figura 3b). Teniendo en cuenta lo analizado, con las condiciones propuestas para los métodos CMS y GCS, no se efectúan cambios dimensionales y de forma en las partículas de HAP luego de ser procesadas. La mayor uniformidad en los tamaños y forma de la HAP sintética empleada en CGS, muestran mayor isotropía de distribución, tamaño y forma de la estructura Ti-HAP-Poros que las obtenidas con HAP bobina y proceso CMS. Además, la forma con factor próximo a esferas de las partículas de HAP sintéticas evitan la acumulación de tensiones en aristas vivas, como las que poseen las partículas de HAP bovina; observadas en la estructura metalográfica por presencia de microfisuras que inician en las aristas de las partículas de HAP bobina.

El análisis químico mediante espectroscopía de dispersión de energía de rayos X de las partículas de HAP bovina y sintética previas al sinterizado arrojaron valores de Ca/P promedio de 1,58 para la HAP bobina empleada en CMS y 2,13 para la HAP utilizada en GCS. Una vez sinterizadas las muestras, se obtuvo una relación Ca/P de 1,61 en CMS y 2,28 para la empleada en GCS, lo que demuestra una escaza descomposición química de las partículas de HAP en ambos casos.

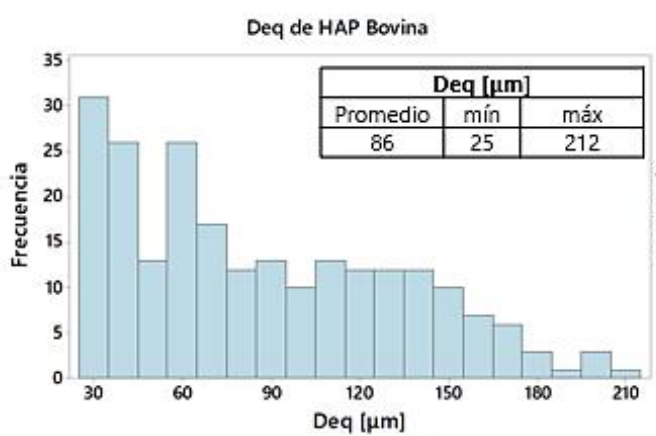

Deq de HAP Sintética

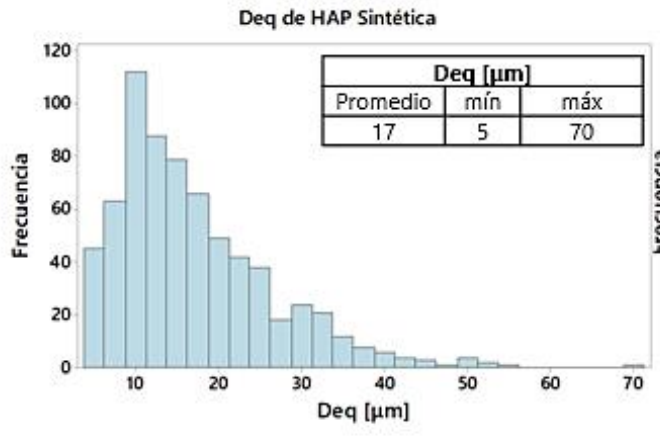

Figura 3: Análisis de tamaño (Deq), forma (FF) e histogramas de distribución de las partículas de HAP bovina y sintética utilizadas.

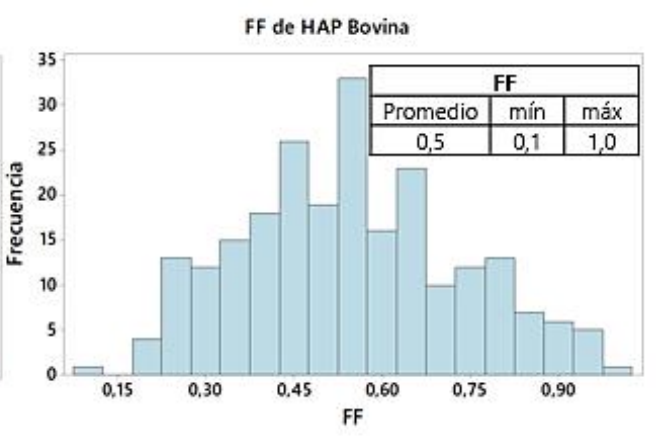

a)

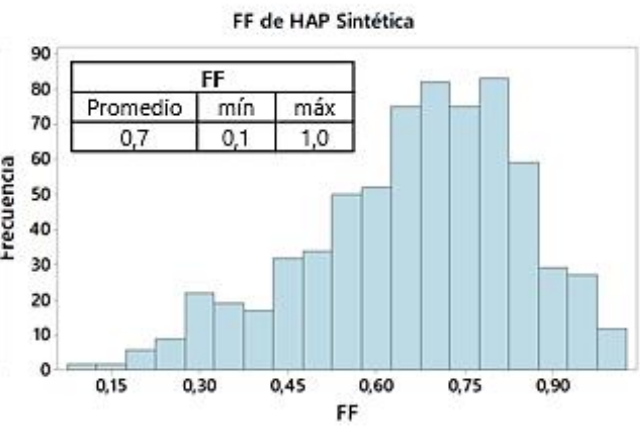

b)
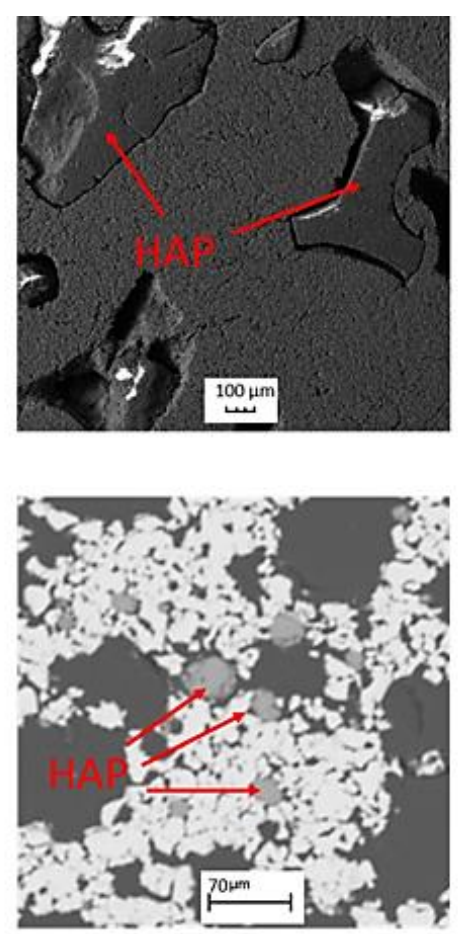

\section{Conclusiones}

Se estudiaron dos procesos pulvimetalúrgicos diferentes y se evaluaron características metalográficas y químicas para cada uno de ellos, permitiendo extraer las siguientes conclusiones:

- Ambos métodos permiten obtener muestras en verde con la suficiente resistencia mecánica para mantener su geometría y ser manipuladas hasta la etapa de sinterización.

- Los parámetros utilizados en los procesos de sinterización CMS y GCS son adecuados para obtener una matriz de $\operatorname{Ti}(\alpha)$. Sin embargo, la forma de las partículas de HAP bobina empleadas en el proceso CMS introducen tensiones que se traducen en microfisuras en la microestructura. 
- Las partículas de HAP bovinas y sintéticas, no se descomponen por difusión o reacción química luego del proceso de sinterización.

- Las partículas de HAP sintéticas presentan un tamaño y forma más estable que las partículas de HAP bovinas, presentando factores de forma y tamaños más adecuados para obtener estructuras homogéneas.

- Mediante el método GCS se pueden obtener estructuras con porosidad controlada. Sin embargo, se deben continuar estudiando la posibilidad de fabricar estructuras con tamaño de poros recomendados para una correcta osteointegración.

\section{Referencias}

Bram, M., Schiefer, H., Bogdanski, D., Köller, M., Buchkremer, H. P., \& Stöver, D. (2006). Implant surgery: How bone bonds to PM titanium. Metal Powder Report, 61(2), 26-28. https://doi.org/10.1016/S0026-0657(06)70603-8

Cantero, S. M., \& Roure, C. A. (2017). Fabricación y caracterización de estructuras de titanio porosas. Universidad Tecnológica Nacional Facultad Regional Córdoba.

Colazo, J., \& Bocco, F. (2017). Desarrollo de biomateriales base titanio activados con hidroxiapatita. Universidad Tecnológica Nacional Facultad Regional Córdoba.

Cui, C., Liu, H., Li, Y., Sun, J., Wang, R., Liu, S., \& Greer, A. L. (2005). Fabrication and biocompatibility of nanoTiO2/titanium alloys biomaterials. Materials Letters, 59(24-25), 3144-3148.

https://doi.org/10.1016/j.matlet.2005.05.037

Esen, Z., \& Bor, S. (2007). Processing of titanium foams using magnesium spacer particles. Scripta Materialia, 56(5), 341344. https://doi.org/10.1016/j.scriptamat.2006.11.010

Lascano, S. (2012). Obtención y caracterización de Ti c.p. poroso para aplicaciones biomédicas.

Murphy, C. M., \& O’Brien, F. J. (2010). Understanding the effect of mean pore size on cell activity in collagenglycosaminoglycan scaffolds. Cell Adhesion and Migration, 4(3), 377-381. https://doi.org/10.4161/cam.4.3.11747

Navarro, M., Michiardi, A., Castaño, O., \& Planell, J. A. (2008). Biomaterials in orthopaedics. Journal of the Royal Society Interface, 5(27), 1137-1158. https://doi.org/10.1098/rsif.2008.0151

Niespodziana, K., Jurczyk, K., Jakubowicz, J., \& Jurczyk, M. (2010). Fabrication and properties of titanium-hydroxyapatite nanocomposites. Materials Chemistry and Physics, 123(1), 160-165.

https://doi.org/10.1016/j.matchemphys.2010.03.076

Niinomi, M. (2008). Mechanical biocompatibilities of titanium alloys for biomedical applications. Journal of the Mechanical Behavior of Biomedical Materials, 1(1), 30-42. https://doi.org/10.1016/j.jmbbm.2007.07.001

Oldani, C. (2014). El camino del titanio como material de reemplazo óseo. Revista de La Facultad de Ciencias Exactas, Físicas y Naturales, 1(1), 67. https://revistas.unc.edu.ar/index.php/FCEFyN/article/view/6969/8046

Rack, H. J., \& Qazi, J. I. (2006). Titanium alloys for biomedical applications. Materials Science and Engineering C, 26(8), 1269-1277. https://doi.org/10.1016/j.msec.2005.08.032

Roure, C. A., Cantero, S. M., Lucci, R. O., Giménez, G. A., \& Padilla, R. L. (2016). Development of porous structures for titanium-based implants. Journal of Chemical Information and Modeling.

https://doi.org/10.1017/CB09781107415324.004

Shettlemore, M. G., \& Bundy, K. J. (1999). Toxicity measurement of orthopedic implant alloy degradation products using a bioluminescent bacterial assay. Journal of Biomedical Materials Research, 45(4), 395. https://doi.org/10.1002/(sici)1097-4636(19990615)45:4<395::aid-jbm15>3.3.co;2-8

Tengvall, P., Lundström, I., Sjöqvist, L., Elwing, H., \& Bjursten, L. M. (1989). Titanium-hydrogen peroxide interaction: model studies of the influence of the inflammatory response on titanium implants. Biomaterials, 10(3), 166-175. https://doi.org/10.1016/0142-9612(89)90019-7 